\title{
A system for miRNAs and cell-free RNA isolation from body fluids
}

Ana Carrasco and Eduard Gallardo, Neuromuscular Diseases Unit, Neurology Department, Hospital de la Santa Creu I Sant Pau, Universitat Autònoma de Barcelona, Institut de Recerca Sant Pau, (Barcelona) and Biomedical Network Research Centre on Rare Diseases (CIBERER), Spain.

David Navarro, DanaGen-BioTed S.L, Barcelona, SPAIN david@danagen.es

\section{Introduction}

miRNAs are small non-coding RNAs about 21-25 nucleotides in length. They are involved in RNA silencing and post-transcriptional regulation of gene expression and they have been found altered in the progression of different diseases. These molecules are found in most body fluids among them plasma and serum.

We quantified three different microRNAs. miR-223-3p has been detected in different experiments in our laboratory and is expressed in control samples at low CT values (around 20). miR-23a-3p and miR-451 are used to monitor sample hemolysis. miR-23a-3p CT value minus miR-451 CT value must be lower than 7 otherwise it is recommended not to use the sample.

DANAGEN-BIOTED has developed a system for isolation of microRNAs and cell-free RNA (cfRNA) for $300 \mu$ l (up to $600 \mu \mathrm{l}$ multiple loading) of plasma or serum using Spin columns that bound RNA with $<1000$ nucleotides. The aim of the study was to compare different methods of microRNAs isolation.

\section{Material and Methods}

\section{microRNA isolation}

The plasma sample was collected from each patient and miRNAs were isolated using 4 different protocols: from $300 \mu \mathrm{l}$ of sample with DANAGENE microRNA and Cell-free RNA Kit with and without carrier (MS2 RNA, Roche) and 200 or $300 \mu l$ of sample using a competitor kit and carrier MS2 .

Universal reverse transcription and real-time PCR amplification

We use the miRCURY LNA'M Universal RT microRNA PCR (EXIQON). This protocol is based on universal reverse transcription (RT) followed by real-time PCR amplification using SYBR-Green. We used LNA ${ }^{\mathrm{TM}}$ enhanced primers specific for the three microRNAs. All reactions were performed in triplicate using a 7900HT device from Applied Biosystems.

\section{Results}

microRNAs were quantified by real-time PCR. The mean of the three threshold cycles $(\mathrm{Ct}$ ) in each condition are shown in Figure 1. For all the miRNAs tested the Ct value was lower with the DANAGEN kit using the same sample volume. Ct values using the DANAGEN kit improved when a carrier was added to the sample.

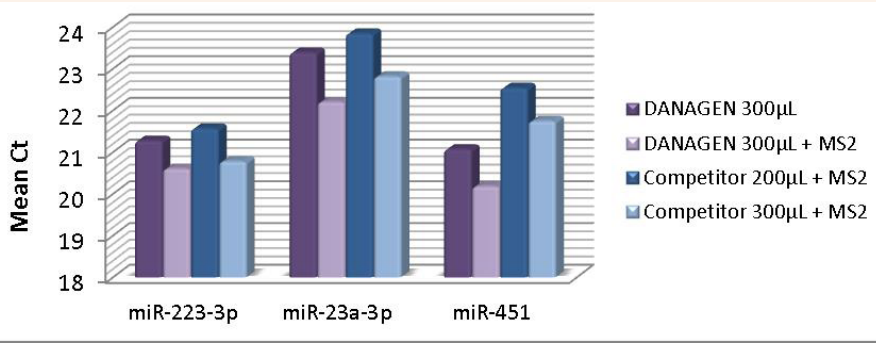

Figure 1. Mean $\mathrm{Ct}$ of 3 different reactions in each condition: Danagen kit w/o

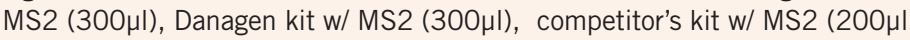
and $300 \mu \mathrm{l})$

\section{Conclusion}

This quantitative analysis of plasma miRNAs shows better results using DANAGENE microRNA and Cell-free RNA Kit. The results are further improved when using a carrier (MS2).

In this paper we demonstrate that our kit can be used for an efficient isolation of small RNA from body fluids, useful for investigating miRNAs and cell-free RNA as circulating biomarkers for cancer and others diseases. 